Mathematical Modelling and Analysis

Volume 18 Number 3, June 2013, 446-460

http://dx.doi.org/10.3846/13926292.2013.807317

(c) Vilnius Gediminas Technical University, 2013
Publisher: Taylor\&Francis and VGTU

http://www.tandfonline.com/TMMA

Print ISSN: 1392-6292

Online ISSN: 1648-3510

\title{
Thin Plate Splines for Transfinite Interpolation at Concentric Circles
}

\section{Aurelian Bejancu}

\section{Kuwait University}

PO Box 5969, 13060 Safat, Kuwait

E-mail: aurelian@sci.kuniv.edu.kw

Received October 7, 2012; revised May 16, 2013; published online June 1, 2013

\begin{abstract}
We propose a new method for constructing a polyspline on annuli, i.e. a $C^{2}$ surface on $\mathbb{R}^{2} \backslash\{0\}$, which is piecewise biharmonic on annuli centered at 0 and interpolates smooth data at all interface circles. A unique surface is obtained by imposing Beppo Levi conditions on the innermost and outermost annuli, and one additional restriction at 0 : either prescribing an extra data value, or asking that the surface is non-singular. We show that the resulting Beppo Levi polysplines on annuli are in fact thin plate splines, i.e. they minimize Duchon's bending energy.
\end{abstract}

Keywords: approximation, interpolation, spline.

AMS Subject Classification: 41A05; 41A15; 41A63.

\section{Introduction}

Thin plate spline interpolation to scattered data was formulated by Duchon [8] as the problem of minimizing the integral

$$
\iint_{\mathbb{R}^{2}}\left(\left|F_{x x}\right|^{2}+2\left|F_{x y}\right|^{2}+\left|F_{y y}\right|^{2}\right) \mathrm{d} x \mathrm{~d} y
$$

among all generalized functions $F$ with second order partial derivatives in $L^{2}\left(\mathbb{R}^{2}\right)$ and taking prescribed values at a finite number of scattered locations. The unique minimizer surface has a convenient radial basis representation with translates of the fundamental solution of the biharmonic operator in $\mathbb{R}^{2}$.

For the problem of surface interpolation of smooth data at given curves within a bounded domain $\Omega$-in CAGD jargon: transfinite interpolation (Sabin [16]) - Kounchev's polyspline method [13] defines a unique piecewise biharmonic $C^{2}$ surface passing through the curve data and satisfying certain boundary conditions. Such a polyspline surface minimizes

$$
\iint_{\Omega}\left|F_{x x}+F_{y y}\right|^{2} \mathrm{~d} x \mathrm{~d} y
$$


subject to the interpolation and boundary conditions.

Let $\mathbb{T}=[-\pi, \pi]$ and assume that $F(x, y)$ is $2 \pi$-periodic in $y$ and its values are prescribed at a finite number of parallel segments $\left\{x_{j}\right\} \times \mathbb{T}$ with $x_{1}<\cdots<x_{n}$. In [5], Bejancu constructed a $C^{2}$ biharmonic polyspline on strips by replacing usual boundary conditions with the Beppo Levi conditions:

$$
F_{x x}, F_{x y}, F_{y y} \in L^{2}(\mathbb{R} \times \mathbb{T}) .
$$

For sufficiently smooth data curves, the resulting polyspline was proved to be in fact a thin plate spline for transfinite interpolation, in the sense that it minimizes integral (1.1) evaluated over the infinite cylinder domain $\mathbb{R} \times \mathbb{T}$, among all interpolants $F \in C^{2}(\mathbb{R} \times \mathbb{T})$ with second partial derivatives in $L^{2}(\mathbb{R} \times \mathbb{T})$.

In the present paper, we obtain a new Beppo Levi polyspline surface designed for transfinite interpolation of smooth data at a finite number of concentric circles. Expressing Duchon's integral (1.1) in polar coordinates, we find that the second partial derivatives of the surface may in this case develop a singularity at the center of the domain circles. In effect, the Beppo Levi conditions together with the transfinite interpolation conditions are not sufficient anymore to determine a unique polyspline on annuli. Instead, we prove (Theorem 1) that uniqueness is achieved by augmenting these conditions with one more restriction imposed at the center of the circles: either prescribing one extra data value, or asking that the surface is biharmonic (and so removing the singularity). We also show (Theorem 2) that, under each additional restriction, such a Beppo Levi polyspline on annuli is in fact a thin plate spline.

The construction of polyspline surfaces on annuli is based on separation of variables, as indicated in [13]. In Section 3, we show that applying this process to a Beppo Levi polyspline leads to a new class of univariate $L$-splines with adjoint end conditions, and we also obtain a key estimate (Theorem 4) required in the construction of Beppo Levi polysplines. The main results, Theorems 1 and 2 stated in Section 2, are proved in Sections 4 and 5, respectively.

Note that Beppo Levi conditions for polysplines were first formulated in [4] in the context of semicardinal interpolation to smooth data at an infinite set of parallel hyperplanes. Related, but different, interpolation problems and approaches appear in $[2,10]$, and their references.

\section{Beppo Levi Polysplines on Annuli}

In this section, we use polar coordinates to formulate the definition of biharmonic Beppo Levi polysplines on annuli and we state the main results of the paper concerning the existence, uniqueness and variational characterization of such polysplines.

\subsection{Beppo Levi conditions in polar coordinates}

Functions of $(x, y)$ will be denoted by uppercase letters such as $S, F, G$, and their form in polar coordinates by the corresponding lowercase letter, as in

$$
F(x, y)=F(r \cos \theta, r \sin \theta)=f(r, \theta), \quad r \geq 0, \theta \in \mathbb{T} .
$$


As is customary, all pairs $(0, \theta)$ are topologically identified with the origin 0 of the plane. Assuming that $F \in C^{2}\left(\mathbb{R}^{2} \backslash\{0\}\right)$, so $f \in C^{2}((0, \infty) \times \mathbb{T})$, we have the following chain relations:

$$
\begin{aligned}
& F_{x x}=\left(\cos ^{2} \theta\right) f_{r r}+\left(\sin ^{2} \theta\right)\left(\frac{1}{r} f_{r}+\frac{1}{r^{2}} f_{\theta \theta}\right)+(\sin 2 \theta)\left(\frac{1}{r^{2}} f_{\theta}-\frac{1}{r} f_{r \theta}\right), \\
& F_{y y}=\left(\sin ^{2} \theta\right) f_{r r}+\left(\cos ^{2} \theta\right)\left(\frac{1}{r} f_{r}+\frac{1}{r^{2}} f_{\theta \theta}\right)-(\sin 2 \theta)\left(\frac{1}{r^{2}} f_{\theta}-\frac{1}{r} f_{r \theta}\right), \\
& F_{x y}=\frac{1}{2}(\sin 2 \theta) f_{r r}-\frac{1}{2}(\sin 2 \theta)\left(\frac{1}{r} f_{r}+\frac{1}{r^{2}} f_{\theta \theta}\right)-(\cos 2 \theta)\left(\frac{1}{r^{2}} f_{\theta}-\frac{1}{r} f_{r \theta}\right) .
\end{aligned}
$$

In particular, these imply the well-known expression of the Laplace operator in polar coordinates:

$$
\Delta F=F_{x x}+F_{y y}=f_{r r}+\frac{1}{r} f_{r}+\frac{1}{r^{2}} f_{\theta \theta} .
$$

Lemma 1. If $F \in C^{2}\left(\mathbb{R}^{2} \backslash\{0\}\right)$, the (Cartesian) Beppo Levi conditions $F_{x x}$, $F_{x y}, F_{y y} \in L^{2}\left(\mathbb{R}^{2} \backslash\{0\}\right)$ hold if and only if, in polar coordinates,

$$
r^{1 / 2} f_{r r}, \frac{1}{r^{1 / 2}} f_{r}+\frac{1}{r^{3 / 2}} f_{\theta \theta}, \frac{1}{r^{3 / 2}} f_{\theta}-\frac{1}{r^{1 / 2}} f_{r \theta} \in L^{2}((0, \infty) \times \mathbb{T}) .
$$

Proof. Sufficiency is a straightforward consequence of the change of variables formula for double integrals in polar coordinates and the fact that all trigonometric coefficients multiplying the functions $f_{r r}, \frac{1}{r} f_{r}+\frac{1}{r^{2}} f_{\theta \theta}$, and $\frac{1}{r^{2}} f_{\theta}-\frac{1}{r} f_{r \theta}$ in the chain identities are uniformly bounded. Necessity follows in the same way, after inverting the system (2.1) to express the above three functions in terms of $F_{x x}, F_{y y}, F_{x y}$ and noting that the $3 \times 3$ inverse matrix of coefficients is actually the same with the original one.

Denote by $V_{2}$ the vector space of functions $F \in C^{2}\left(\mathbb{R}^{2} \backslash\{0\}\right) \cap C\left(\mathbb{R}^{2}\right)$ that satisfy the Beppo Levi conditions of Lemma 1 . For any $F, G \in V_{2}$, using the chain relations (2.1), we are led to the following polar expressions of Duchon's semi-inner product and induced seminorm (1.1) on $V_{2}$ :

$$
\begin{aligned}
\langle f, g\rangle_{V_{2}}= & \int_{0}^{\infty} \int_{-\pi}^{\pi}\left\{f_{r r} \bar{g}_{r r}+2\left[\frac{f_{\theta}}{r^{2}}-\frac{f_{r \theta}}{r}\right]\left[\frac{\bar{g}_{\theta}}{r^{2}}-\frac{\bar{g}_{r \theta}}{r}\right]\right. \\
& \left.+\left[\frac{f_{\theta \theta}}{r^{2}}+\frac{f_{r}}{r}\right]\left[\frac{\bar{g}_{\theta \theta}}{r^{2}}+\frac{\bar{g}_{r}}{r}\right]\right\} r \mathrm{~d} \theta \mathrm{d} r \\
\|f\|_{V_{2}}^{2}= & \int_{0}^{\infty} \int_{-\pi}^{\pi}\left\{\left|f_{r r}\right|^{2}+2\left|\frac{f_{\theta}}{r^{2}}-\frac{f_{r \theta}}{r}\right|^{2}+\left|\frac{f_{\theta \theta}}{r^{2}}+\frac{f_{r}}{r}\right|^{2}\right\} r \mathrm{~d} \theta \mathrm{d} r .
\end{aligned}
$$

Note that $V_{2}$ is a subspace of the Beppo Levi space $\mathrm{BL}_{2}\left(\mathbb{R}^{2}\right)$ of order 2 over $\mathbb{R}^{2}$ (see [18, Definition 10.37]).

\subsection{Main results}

Given the radii $r_{0}=0<r_{1}<\cdots<r_{n}<\infty=r_{n+1}$, let $\rho=\left\{r_{1}, \ldots, r_{n}\right\}$ and define the open annuli

$$
\Omega_{j}=\left\{(r, \theta) \in(0, \infty) \times \mathbb{T}: r_{j}<r<r_{j+1}\right\}, \quad j \in\{0,1, \ldots, n\} .
$$


Note that $\Omega_{0}$ is a punctured open disk.

Definition 1. A function $S: \mathbb{R}^{2} \rightarrow \mathbb{R}$ is called a biharmonic Beppo Levi $(B L)$ polyspline on annuli determined by $\rho$ if the following conditions hold:

(i) $S$ is biharmonic on each annulus $\Omega_{j}, j \in\{0,1, \ldots, n\}$ :

$$
\Delta^{2} S(x, y)=\left(\partial_{r r}+\frac{1}{r} \partial_{r}+\frac{1}{r^{2}} \partial_{\theta \theta}\right)^{2} s(r, \theta)=0, \quad \forall(r, \theta) \in \Omega_{j} ;
$$

(ii) $S \in C^{2}\left(\mathbb{R}^{2} \backslash\{0\}\right) \cap C\left(\mathbb{R}^{2}\right)$;

(iii) the polar form $s$ of $S$ satisfies (2.2) in place of $f$.

The vector space of all biharmonic BL polysplines on annuli determined by $\rho$ will be denoted by $\mathcal{S}_{B L_{2}}(\rho)$. Note that $\mathcal{S}_{B L_{2}}(\rho) \subset V_{2}$.

Let $W^{2}$ be the 'weighted' Wiener algebra [7, p. 222] of all continuous periodic functions $\mu: \mathbb{T} \rightarrow \mathbb{C}$ such that

$$
\|\mu\|_{W^{2}}:=\sum_{k=-\infty}^{\infty}\left|\widehat{\mu}_{k}\right|(1+|k|)^{2}<\infty
$$

where $\widehat{\mu}_{k}:=\frac{1}{2 \pi} \int_{-\pi}^{\pi} e^{-i k \theta} \mu(\theta) \mathrm{d} \theta$, for $k \in \mathbb{Z}$, are the Fourier coefficients of $\mu$. Note that $C^{3}(\mathbb{T}) \subset W^{2} \subset C^{2}(\mathbb{T})$. For data functions in $W^{2}$ along the concentric circles $r=r_{j}$, the first main result below establishes the existence and uniqueness of solutions for two types of transfinite interpolation problems with biharmonic BL polysplines on annuli.

Theorem 1. Let $\rho$ be a given set of $n$ radii as above and let $\mu_{j} \in W^{2}, j \in$ $\{1, \ldots, n\}$, be $n$ data functions.

(a) For any real value $\alpha$, there exists a unique biharmonic BL polyspline $S^{A} \in \mathcal{S}_{B L_{2}}(\rho)$, of polar form $s^{A}$, satisfying

$$
\left\{\begin{array}{l}
s^{A}\left(r_{j}, \theta\right)=\mu_{j}(\theta), \quad \forall \theta \in \mathbb{T}, \forall j \in\{1, \ldots, n\}, \\
S^{A}(0)=\alpha
\end{array}\right.
$$

(b) There exists a unique biharmonic BL polyspline $S^{B} \in \mathcal{S}_{B L_{2}}(\rho)$ such that $S^{B}$ is biharmonic at 0 and its polar form $s^{B}$ satisfies the transfinite equations

$$
s^{B}\left(r_{j}, \theta\right)=\mu_{j}(\theta), \quad \forall \theta \in \mathbb{T}, \forall j \in\{1, \ldots, n\} .
$$

To the author's knowledge, a polyspline satisfying both finite and transfinite interpolation conditions as in part (a) above has not appeared before in the literature. Kounchev [13, Ch. 8 \& 9] has previously defined polysplines "supported in the annulus" $r_{1} \leq r \leq r_{n}$, and polysplines "supported in the ball" $0 \leq r \leq r_{n}$. The latter possess a biharmonic piece on the whole inner disk $0 \leq r<r_{1}$, as in part (b). However, while Kounchev's polysplines satisfy classical Dirichlet boundary conditions on the outer circle $r=r_{n}$ (and on the inner circle $r=r_{1}$ if "supported in the annulus"), our automatic Beppo Levi conditions are imposed on the unbounded annulus $r>r_{n}$ and on the punctured disk $0<r<r_{1}$. 
Theorem 2. Consider the biharmonic BL polysplines $S^{A}$ and $S^{B}$ obtained in Theorem 1. Then we have $\left\|s^{A}\right\|_{V_{2}}<\|f\|_{V_{2}}$ for any other function $F \in V_{2}$ satisfying (2.5) in place of $S^{A}$. Also, if any other function $F \in V_{2}$ satisfies the transfinite conditions (2.6) in place of $S^{B}$, then $\left\|s^{B}\right\|_{V_{2}}<\|f\|_{V_{2}}$.

The last result shows that the biharmonic BL polysplines $S^{A}$ and $S^{B}$ are genuine thin plate splines for transfinite interpolation at the given concentric circles. Theorems 1 and 2 are proved in Sections 4 and 5, using Fourier series representations of $S^{A}$ and $S^{B}$. The necessary conditions for the amplitude coefficients of these representations are derived in Section 3.

Remark 1. The condition that the data functions belong to the Wiener-type algebra $W^{2}$ is by no means necessary; rather, it is an assumption required by our constructive proof of Theorem 1, based on Fourier series estimates in terms of the norm (2.4). It remains a question for further research whether more general biharmonic $\mathrm{BL}$ polysplines can be constructed to interpolate traces of arbitrary Beppo Levi surfaces from $\mathrm{BL}_{2}\left(\mathbb{R}^{2}\right)$.

In comparison, Kounchev's existence results [13, pp. 446-453] on $C^{2}$ biharmonic polyspline interpolation with Dirichlet boundary conditions are obtained via the method of frozen coefficients for elliptic boundary value problems, which requires data functions from the fractional Sobolev space $H^{7 / 2}(\mathbb{T})$. We note that $H^{7 / 2}(\mathbb{T}) \subset H^{3}(\mathbb{T}) \subset W^{2}$, the first inclusion being well known. The second inclusion follows from the fact that, if $\widehat{\mu}_{k}, k \in \mathbb{Z}$, denote the Fourier coefficients of $\mu \in H^{3}(\mathbb{T})$, then

$$
2 \sum_{k \neq 0} k^{2}\left|\widehat{\mu}_{k}\right| \leq \sum_{k \neq 0} k^{6}\left|\widehat{\mu}_{k}\right|^{2}+\sum_{k \neq 0} k^{-2}<\infty .
$$

These observations are also directly relevant to the applicability of biharmonic polysplines in the typical situation in which the interpolated curves are periodic cubic splines generated from discrete data at points along the concentric circles. Indeed, any periodic cubic spline on $\mathbb{T}$ belongs to $H^{3}(\mathbb{T})$, hence our results guarantee the existence of biharmonic BL polysplines through cubic spline curves given at the concentric circles. However, as Kounchev noted, periodic cubic splines fail to belong to $H^{7 / 2}(\mathbb{T})$ and must be replaced by higher degree splines as data curves in his results.

\section{Separation of Variables}

\section{$3.1 \quad L$-spline Fourier coefficients}

For any $F \in V_{2}$ and $r>0$, the Fourier coefficients of $f(r, \theta)$ with respect to $\theta$ are defined by

$$
\widehat{f_{k}}(r):=\frac{1}{2 \pi} \int_{-\pi}^{\pi} e^{-i k \theta} f(r, \theta) \mathrm{d} \theta, \quad k \in \mathbb{Z} .
$$

For any $k \in \mathbb{Z}$, note that $\widehat{f}_{k} \in C^{2}(0, \infty)$ and $\widehat{f}_{k}$ is continuous at $r=0$, since $f \in C^{2}((0, \infty) \times \mathbb{T})$ and $F$ is continuous at 0 . The effect of the Beppo Levi conditions on these parameter-dependent Fourier coefficients is displayed in the following result, which was also obtained in [1] by different arguments. 
Lemma 2. If $F \in V_{2}$, then, as functions of $r$, the Fourier coefficients (3.1) satisfy the conditions:

$$
\begin{gathered}
r^{1 / 2} \frac{\mathrm{d}^{2}}{\mathrm{~d} r^{2}} \widehat{f}_{0} \quad \text { and } \quad r^{-1 / 2} \frac{\mathrm{d}}{\mathrm{d} r} \widehat{f}_{0} \in L^{2}(0, \infty) ; \\
r^{1 / 2} \frac{\mathrm{d}^{2}}{\mathrm{~d} r^{2}} \widehat{f}_{k} \quad \text { and } \quad r^{-1 / 2} \frac{\mathrm{d}}{\mathrm{d} r} \widehat{f}_{k}-r^{-3 / 2} \widehat{f}_{k} \in L^{2}(0, \infty), \quad \text { if }|k|=1 ; \\
r^{1 / 2} \frac{\mathrm{d}^{2}}{\mathrm{~d} r^{2}} \widehat{f}_{k}, r^{-1 / 2} \frac{\mathrm{d}}{\mathrm{d} r} \widehat{f}_{k} \quad \text { and } \quad r^{-3 / 2} \widehat{f}_{k} \in L^{2}(0, \infty), \quad \text { if }|k| \geq 2
\end{gathered}
$$

Proof. The conclusion is a direct consequence of Plancherel's formula:

$$
\frac{1}{2 \pi}\|f\|_{V_{2}}^{2}=\sum_{k=-\infty}^{\infty} \int_{0}^{\infty}\left\{\left|\frac{\mathrm{d}^{2} \widehat{f}_{k}}{\mathrm{~d} r^{2}}\right|^{2}+2 k^{2}\left|\frac{\widehat{f}_{k}}{r^{2}}-\frac{1}{r} \frac{\mathrm{d} \widehat{f}_{k}}{\mathrm{~d} r}\right|^{2}+\left|k^{2} \frac{\widehat{f}_{k}}{r^{2}}-\frac{1}{r} \frac{\mathrm{d} \widehat{f}_{k}}{\mathrm{~d} r}\right|^{2}\right\} r \mathrm{~d} r .
$$

This, in turn, is established in the same way as Eq. (27) in [5, Lemma 3.5], based on the smoothness of $f$ and the Beppo Levi conditions (2.2).

We now look at necessary conditions for the Fourier coefficients of a biharmonic BL polyspline $S$ from Definition 1 . For each $j \in\{0,1, \ldots, n\}$, since $S$ is biharmonic, hence analytic, in the open annulus $\Omega_{j}$, its polar form $s$ admits an absolutely convergent Fourier representation

$$
s(r, \theta)=\sum_{k=-\infty}^{\infty} \widehat{s}_{k}(r) e^{i k \theta}, \quad(r, \theta) \in \Omega_{j}
$$

Applying the bi-Laplacean in polar coordinates, by permitted differentiation we obtain, for all $(r, \theta) \in \Omega_{j}$,

$$
0=\left(\partial_{r r}+\frac{1}{r} \partial_{r}+\frac{1}{r^{2}} \partial_{\theta \theta}\right)^{2} s(r, \theta)=\sum_{k=-\infty}^{\infty}\left(\frac{\mathrm{d}^{2}}{\mathrm{~d} r^{2}}+\frac{1}{r} \frac{\mathrm{d}}{\mathrm{d} r}-\frac{k^{2}}{r^{2}}\right)^{2}\left[\widehat{s}_{k}(r)\right] e^{i k \theta} .
$$

Since all coefficients of the last Fourier series must vanish, letting

$$
L_{k}:=r\left(\frac{\mathrm{d}^{2}}{\mathrm{~d} r^{2}}+\frac{1}{r} \frac{\mathrm{d}}{\mathrm{d} r}-\frac{k^{2}}{r^{2}}\right)^{2}
$$

it follows that, for each $k \in \mathbb{Z}$, we have

$$
L_{k}\left[\widehat{s}_{k}(r)\right]=0, \quad \forall r \in(0, \infty) \backslash\left\{r_{1}, \ldots, r_{n}\right\} .
$$

Therefore $\widehat{s}_{k}$ is a univariate $L_{k}$-spline, in the sense that it is piecewise in the null-space of $L_{k}$ and its pieces are joined with $C^{2}$ smoothness at each knot $r_{j}, 1 \leq j \leq n$. Note that the factor $r$ was inserted in (3.3) so that $L_{k}$ becomes a formally self-adjoint operator. This agrees with the general definition of $L$-splines proposed by Lucas [14], who considered non-singular self-adjoint operators $L$ with variable coefficients, as well as with the extended definition of Jerome and Pierce [11], who treated singular such operators. However, these 
generalized $L$-splines were defined on a compact interval, while in our setting $\widehat{s}_{k}$ is defined on $[0, \infty)$.

Further, it is known (e.g. [13]) that the null-space of $L_{k}$ is

$$
\operatorname{Ker} L_{k}= \begin{cases}\operatorname{span}\left\{r^{2}, r^{2} \ln r, 1, \ln r\right\}, & \text { if } k=0, \\ \operatorname{span}\left\{r^{3}, r, r \ln r, r^{-1}\right\}, & \text { if }|k|=1, \\ \operatorname{span}\left\{r^{|k|+2}, r^{|k|}, r^{-|k|+2}, r^{-|k|}\right\}, & \text { if }|k| \geq 2 .\end{cases}
$$

Hence a straightforward evaluation of relevant integrals for each element of this four-dimensional null-space shows the precise effect of the conditions of Lemma 2 on the extreme pieces of such a $L_{k}$-spline, as follows.

Corollary 1 [Al-Sahli [1]]. If $S$ is a biharmonic BL polyspline on annuli determined by $\rho$, then the Fourier coefficients $\widehat{s}_{k}$ of $s$ satisfy:

$$
\begin{aligned}
& \widehat{s}_{0}(r) \in \begin{cases}\operatorname{span}\left\{r^{2}, r^{2} \ln r, 1\right\}, & \text { for } r \in\left(0, r_{1}\right), \\
\operatorname{span}\{1, \ln r\}, & \text { for } r \in\left(r_{n}, \infty\right),\end{cases} \\
& \widehat{s}_{k}(r) \in\left\{\begin{array}{ll}
\operatorname{span}\left\{r^{|k|+2}, r^{|k|}\right\}, & \text { for } r \in\left(0, r_{1}\right), \\
\operatorname{span}\left\{r^{-|k|+2}, r^{-|k|}\right\}, & \text { for } r \in\left(r_{n}, \infty\right),
\end{array} \text { if } k \neq 0 .\right.
\end{aligned}
$$

These conditions can also be expressed in differential form using Euler's substitution $r=e^{v}$, which transforms $L_{k}$ into a differential operator with constant coefficients. We will employ the notations:

$$
\begin{aligned}
G_{0} & :=\frac{\mathrm{d}^{3}}{\mathrm{~d} r^{3}}-\frac{1}{r} \frac{\mathrm{d}^{2}}{\mathrm{~d} r^{2}}+\frac{1}{r^{2}} \frac{\mathrm{d}}{\mathrm{d} r}=\frac{1}{r^{3}}\left(r \frac{\mathrm{d}}{\mathrm{d} r}\right)\left(r \frac{\mathrm{d}}{\mathrm{d} r}-2\right)^{2}, \\
R_{0} & :=\frac{1}{r}\left[\frac{\mathrm{d}^{2}}{\mathrm{~d} r^{2}}+\frac{1}{r} \frac{\mathrm{d}}{\mathrm{d} r}\right]=\frac{1}{r^{3}}\left(r \frac{\mathrm{d}}{\mathrm{d} r}\right)^{2},
\end{aligned}
$$

and, for $k \neq 0$,

$$
\begin{aligned}
G_{k} & :=\frac{1}{r}\left[\frac{\mathrm{d}^{2}}{\mathrm{~d} r^{2}}-\frac{2|k|+1}{r} \frac{\mathrm{d}}{\mathrm{d} r}+\frac{|k|(|k|+2)}{r^{2}}\right] \\
& =\frac{1}{r^{3}}\left(r \frac{\mathrm{d}}{\mathrm{d} r}-|k|\right)\left(r \frac{\mathrm{d}}{\mathrm{d} r}-|k|-2\right), \\
R_{k} & :=\frac{1}{r}\left[\frac{\mathrm{d}^{2}}{\mathrm{~d} r^{2}}+\frac{2|k|-1}{r} \frac{\mathrm{d}}{\mathrm{d} r}+\frac{|k|(|k|-2)}{r^{2}}\right] \\
& =\frac{1}{r^{3}}\left(r \frac{\mathrm{d}}{\mathrm{d} r}+|k|\right)\left(r \frac{\mathrm{d}}{\mathrm{d} r}+|k|-2\right) .
\end{aligned}
$$

Then, for $k \neq 0, G_{k}$ and $R_{k}$ are pairwise formally adjoint, $R_{k}^{*}=G_{k}$, and we have the factorization $L_{k}=G_{k}\left(r^{3} R_{k}\right)$, while, for $k=0$, we have $R_{0}^{*}=$ $\frac{1}{r^{3}}\left(r \frac{\mathrm{d}}{\mathrm{d} r}-2\right)^{2}$ and $L_{0}=R_{0}^{*}\left(r^{3} R_{0}\right)$.

Definition 2. Let $k \in \mathbb{Z}$. The function $\eta:[0, \infty) \rightarrow \mathbb{C}$ is called a Beppo Levi $L_{k}$-spline on $\rho$ if the following conditions hold: 
(i) $L_{k} \eta(r)=0, \forall r \in\left(r_{j}, r_{j+1}\right), \forall j \in\{1, \ldots, n-1\}$;

(ii) $G_{k} \eta(r)=0, \forall r \in\left(0, r_{1}\right)$, and $R_{k} \eta(r)=0, \forall r>r_{n}$;

(iii) $\eta$ is $C^{2}$-continuous at each node $r_{1}, \ldots, r_{n}$.

The class of all Beppo Levi $L_{k}$-splines on $\rho$ will be denoted by $\mathcal{S}_{k}(\rho)$.

Note that, if $\eta \in \mathcal{S}_{k}(\rho)$, then $\eta$ is necessarily right-continuous at $r=0$.

Remark 2. For $k \neq 0$, the restriction of a Beppo Levi $L_{k}$-spline to the compact interval $\left[r_{1}, r_{n}\right]$ will inherit, by continuity, the conditions of part (ii) as endpoint conditions at $r_{1}$ and $r_{n}$. The peculiar property that, for $k \neq 0,\left(G_{k}, R_{k}\right)$ is a pair of formally adjoint operators in (ii) mirrors the similar property formulated in [5, Def. 1.2] for a class of exponential $L$-splines generated as Fourier coefficients of Beppo Levi polysplines on strips. This contrasts the literature on generalized $L$-splines in which 'natural' end conditions are usually formulated by means of one and the same differential operator at both endpoints [17].

As the above arguments show, if $S \in \mathcal{S}_{B L_{2}}(\rho)$, then $\widehat{s}_{k} \in \mathcal{S}_{k}(\rho), \forall k \in \mathbb{Z}$. Conversely, the construction and variational characterization of biharmonic BL polysplines in the last two sections of the paper are based on the existence and uniqueness of interpolation from the spaces $\mathcal{S}_{k}(\rho), k \in \mathbb{Z}$. The latter results, which do not follow from the known theory of generalized $L$-splines due to the new type of end conditions on the infinite domain $[0, \infty)$, are established separately in [6] and [3], as stated below.

Theorem 3. Let $\nu_{1}, \ldots, \nu_{n}$ be arbitrary real values.

(i) [6] For each $k \neq 0$, there exists a unique $\sigma \in \mathcal{S}_{k}(\rho)$, such that

$$
\sigma\left(r_{j}\right)=\nu_{j}, \quad j \in\{1, \ldots, n\} .
$$

(ii) [3] Let $k=0$. Given any real number $\alpha$, there exists a unique $\sigma^{A} \in \mathcal{S}_{0}(\rho)$, satisfying both the system (3.6) and the additional interpolation condition $\sigma^{A}(0)=\alpha$. Also, there exists a unique $\sigma^{B} \in \mathcal{S}_{0}(\rho)$ satisfying both (3.6) and the condition that $\sigma^{B} \in \operatorname{span}\left\{r^{2}, 1\right\}$ for $r \in\left(0, r_{1}\right)$.

Note that the Beppo Levi $L_{0}$-splines are in fact profiles of radially symmetric biharmonic polyspline surfaces. In particular, the above non-singular profile $\sigma^{B}$ has first been found by Rabut [15] via the theory of variational splines and reproducing kernels. Moreover, the compactly supported profile $\eta_{2}$ recently constructed by Johnson [12] is also seen to be a Beppo Levi $L_{0}$-spline.

The construction of biharmonic BL polysplines in the next section relies crucially on estimating the dependence of the Beppo Levi $L_{k}$-spline interpolation scheme on the parameter $k$. To describe this result, we need to introduce, for each $k \in \mathbb{Z}$ with $|k| \geq 2$ and each $j \in\{1, \ldots, n\}$, the unique Lagrange function $\chi_{k, j} \in \mathcal{S}_{k}(\rho)$ satisfying the interpolation conditions

$$
\chi_{k, j}\left(r_{j}\right)=1 \quad \text { and } \quad \chi_{k, j}\left(r_{l}\right)=0 \quad \text { for } l \in\{1, \ldots, n\} \backslash\{j\} .
$$


Then the Beppo Levi $L_{k}$-spline $\sigma$ of Theorem 3 (i) admits the representation

$$
\sigma(r)=\sum_{j=1}^{n} \nu_{j} \chi_{k, j}(r), \quad \forall r \in[0, \infty)
$$

Now we can state the following analog of [5, Theorem 2.5].

Theorem 4. There exist an integer $k_{0}=k_{0}(\rho) \geq 3$ and a positive constant $C_{0}=C_{0}\left(n, r_{1}\right)$ such that, for all $j \in\{1, \ldots, n\}$ and $m \in\{0,1,2\}$, we have

$$
\left|\frac{d^{m}}{d r^{m}} \chi_{k, j}(r)\right| \leq C_{0}|k|^{m}, \quad \forall r>0, \forall|k| \geq k_{0} .
$$

Remark 3. Kounchev [13, p. 128 \& Part II] provided estimates similar to (3.9) in the case of cardinal $L$-spline interpolation at all integer nodes, based on B-spline representations. By contrast, in the next subsection, our proof of (3.9) for finitely many non-equidistant nodes is based on representations by translates of positive definite functions with non-compact support.

\subsection{Proof of Theorem 4}

For each $|k| \geq 2$, define

$$
\varphi_{k}(r):=\frac{1}{2} \begin{cases}r^{|k|}\left[(1+|k|)+(1-|k|) r^{2}\right], & 0 \leq r \leq 1 \\ r^{-|k|}\left[(1-|k|)+(1+|k|) r^{2}\right], & 1<r\end{cases}
$$

By construction, $\varphi_{k}$ is $C^{2}$-continuous at $r=1, G_{k} \varphi_{k}(r)=0$ for $0<r<1$, and $R_{k} \varphi_{k}(r)=0$ for $r>1$.

Lemma 3. If $|k| \geq 2$ and $j \in\{1, \ldots, n\}$, there exist unique coefficients $a_{j q}$, $q \in\{1, \ldots, n\}$, such that the Lagrange function $\chi_{k, j} \in \mathcal{S}_{k}(\rho)$ satisfying the interpolation conditions (3.7) admits the representation

$$
\chi_{k, j}(r)=\sum_{q=1}^{n} a_{j q} \varphi_{k}\left(\frac{r}{r_{q}}\right), \quad r \in[0, \infty) .
$$

Proof. We introduce the auxiliary function

$$
\psi_{k}(t):=e^{-t} \varphi_{k}\left(e^{t}\right)=\frac{1}{2} e^{-|k||t|}\left[(1-|k|) e^{-|t|}+(1+|k|) e^{|t|}\right], \quad t \in \mathbb{R},
$$

whose Fourier transform is

$$
\widehat{\psi}_{k}(\tau)=\int_{-\infty}^{\infty} e^{-i t \tau} \psi_{k}(t) \mathrm{d} t=\frac{4|k|\left(k^{2}-1\right)}{\left[(|k|-1)^{2}+\tau^{2}\right]\left[(|k|+1)^{2}+\tau^{2}\right]}, \quad \tau \in \mathbb{R} .
$$

Note that $\psi_{k}$ is a positive definite function, since $\widehat{\psi}_{k}(\tau)>0, \forall \tau \in \mathbb{R}$. Letting $t_{j}:=\ln r_{j}$, it follows that the $n \times n$ matrix

$$
N_{k}:=\left(\psi_{k}\left(t_{j}-t_{q}\right)\right)_{j, q=1}^{n}
$$


is positive definite, hence nonsingular. Therefore there exist unique coefficients $b_{j 1}, \ldots, b_{j n}$, such that the expression

$$
\widetilde{\chi}_{k, j}(t):=\sum_{q=1}^{n} b_{j q} e^{-\left(t_{j}-t_{q}\right)} \varphi_{k}\left(e^{t-t_{q}}\right)
$$

satisfies the interpolation conditions $\widetilde{\chi}_{k, j}\left(t_{j}\right)=1$ and $\tilde{\chi}_{k, j}\left(t_{q}\right)=0$ for $q \neq j$. Let $a_{j q}:=b_{j q} e^{-\left(t_{j}-t_{q}\right)}$ and use the change of variable $r=e^{t}$. Then the righthand side of $(3.11)$ coincides with $\widetilde{\chi}_{k, j}(\ln r)$ and it belongs to $\mathcal{S}_{k}(\rho)$ due to the noted properties of $\varphi_{k}$. The lemma follows from the uniqueness result of Theorem 3.

Now consider the $n \times n$ matrix

$$
M_{k}:=\left(\varphi_{k}\left(\frac{r_{j}}{r_{q}}\right)\right)_{j, q=1}^{n}
$$

of the interpolation system (3.7) under representation (3.11). The proof of Lemma 3 shows that $M_{k}$ is nonsingular for each $|k| \geq 2$. On the other hand, for a fixed $r>0, r \neq 1$, we have $\lim _{|k| \rightarrow \infty} \varphi_{k}(r)=0$. This implies that, as $|k| \rightarrow \infty, M_{k}$ converges to $I$, the identity matrix of order $n$. Hence, there exists an integer $k_{0}=k_{0}(\rho) \geq 2$ such that

$$
\left\|I-M_{k}\right\|_{2} \leq 1 / 2
$$

for all $k \in \mathbb{Z}$ with $|k| \geq k_{0}$, where $\|\cdot\|_{2}$ is the matrix norm induced by the Euclidean norm in $\mathbb{R}^{n}$. Invoking a well-known matrix analysis result $[9$, p. 58], we obtain

$$
\left\|M_{k}^{-1}\right\|_{2} \leq\left(1-\left\|I-M_{k}\right\|_{2}\right)^{-1} \leq 2, \quad \forall|k| \geq k_{0} .
$$

Further, note that, if $\mathbf{e}_{j}$ denotes the $j$-th column of $I$, then

$$
\mathbf{a}_{j}:=\left(a_{j 1}, \ldots, a_{j n}\right)^{T}=M_{k}^{-1} \mathbf{e}_{j}
$$

is the $j$-th column of the inverse matrix $M_{k}^{-1}$. Thus, the coefficients $a_{j q}$ of representation (3.11) satisfy the uniform bound

$$
\left|a_{j q}\right| \leq\left\|\mathbf{a}_{j}\right\|_{2} \leq\left\|M_{k}^{-1}\right\|_{2} \leq 2, \quad \forall j, q \in\{1, \ldots, n\}, \forall|k| \geq k_{0},
$$

where $\left\|\mathbf{a}_{j}\right\|_{2}$ is the Euclidean norm in $\mathbb{R}^{n}$.

Next, elementary calculus shows that, for some $M>0$,

$$
\left\{\begin{array}{l}
0<\varphi_{k}(r) \leq \frac{|k|-1}{|k|-2} \leq 2, \\
\left|\varphi_{k}^{(m)}(r)\right| \leq M|k|^{m}, \quad \text { for } m \in\{1,2\},
\end{array} \quad \forall r>0, \forall|k| \geq 3 .\right.
$$

Therefore (3.11) and (3.12) imply, for all $j \in\{1, \ldots, n\}, m \in\{0,1,2\}$, and $|k| \geq \max \left\{3, k_{0}\right\}$,

$$
\left|\frac{d^{m}}{d r^{m}} \chi_{k, j}(r)\right| \leq \sum_{q=1}^{n}\left|a_{j q}\right|\left|\varphi_{k}^{(m)}\left(\frac{r}{r_{q}}\right)\right| \frac{1}{r_{q}^{m}} \leq C_{0}|k|^{m}, \quad \forall r>0,
$$

where $C_{0}:=2 n r_{1}^{-2} \max \{2, M\}$. This establishes (3.9). 


\section{Construction of Biharmonic BL Polysplines}

In this section, we prove Theorem 1. To establish part (a), we start from the absolutely convergent Fourier series representations

$$
\mu_{j}(\theta)=\sum_{k=-\infty}^{\infty} \widehat{\mu}_{j, k} e^{i k \theta}, \quad \forall \theta \in \mathbb{T}, \forall j \in\{1, \ldots, n\}
$$

where $\widehat{\mu}_{j, k}$, for $k \in \mathbb{Z}$, are the Fourier coefficients of $\mu_{j} \in W^{2}$. For fixed $k \neq 0$, by Theorem $3(\mathrm{i})$, let $\widehat{s}_{k} \in \mathcal{S}_{k}(\rho)$ be the unique Beppo Levi $L_{k}$-spline on $\rho$ satisfying $\widehat{s}_{k}\left(r_{j}\right)=\widehat{\mu}_{j, k}, j \in\{1, \ldots, n\}$. Therefore (3.8) implies

$$
\widehat{s}_{k}(r)=\sum_{j=1}^{n} \widehat{\mu}_{j, k} \chi_{k, j}(r), \quad r \in[0, \infty) .
$$

Also, for $k=0$, denote by $\widehat{s}_{0}^{A}$ the unique Beppo Levi $L_{0}$-spline on $\rho$ satisfying both $\widehat{s}_{0}^{A}\left(r_{j}\right)=\widehat{\mu}_{j, 0}, j \in\{1, \ldots, n\}$, and the additional interpolation condition $\widehat{s}_{0}^{A}(0)=\alpha$, as in Theorem 3 (ii).

Let $s^{A}(r, \theta)$ be defined by

$$
s^{A}(r, \theta):=\widehat{s}_{0}^{A}(r)+\sum_{k \in \mathbb{Z} \backslash\{0\}} \widehat{s}_{k}(r) e^{i k \theta}, \quad(r, \theta) \in[0, \infty) \times \mathbb{T} .
$$

We now show that $s^{A}$ and its version in Cartesian coordinates $S^{A}$ have all the properties required in Theorem 1 (a).

We first claim that the series (4.1) is absolutely and uniformly convergent on compact sets in $[0, \infty) \times \mathbb{T}$ and can be differentiated term-wise for any partial derivative operator of total order $\leq 2$ on $(0, \infty) \times \mathbb{T}$. Indeed, this follows by estimates similar to those of [5, p. 251] (not repeated here, for brevity), based on the stability result (3.9), which is valid for all sufficiently large $|k|$. Therefore $s^{A}$ satisfies the transfinite interpolation conditions on the first row of $(2.5)$ and $s^{A} \in C^{2}((0, \infty) \times \mathbb{T})$. Since all terms of its defining series vanish for $r=0$, except for $\widehat{s}_{0}^{A}(0)=\alpha$, it also follows that $s^{A}$ verifies the additional condition $s^{A}(0, \theta)=\alpha$.

Moreover, each term of the series (4.1) is biharmonic (in Cartesian coordinates) since, for all $(r, \theta) \in \Omega_{j}, j \in\{0,1, \ldots, n\}$, we have

$$
\begin{gathered}
\left(\partial_{r r}+\frac{1}{r} \partial_{r}+\frac{1}{r^{2}} \partial_{\theta \theta}\right)^{2}\left[\widehat{s}_{k}(r) e^{i k \theta}\right]=e^{i k \theta} L_{k} \widehat{s}_{k}(r)=0, \quad \forall k \neq 0, \\
\left(\partial_{r r}+\frac{1}{r} \partial_{r}+\frac{1}{r^{2}} \partial_{\theta \theta}\right)^{2} \widehat{s}_{0}^{A}(r)=L_{0} \widehat{s}_{0}^{A}(r)=0 .
\end{gathered}
$$

Hence, by [5, Lemma 3.4], $S^{A}$ is biharmonic on each open annulus $\Omega_{j}, j \in$ $\{0,1, \ldots, n\}$.

Next, we show that $f:=s^{A}$ satisfies the Beppo Levi conditions (2.2). It will be sufficient to prove that, for each of the three functions appearing in (2.2), its term-wise Fourier expansion derived from (4.1) is a Cauchy series in the $L^{2}$-norm on the extreme annuli $\left(0, r_{1}\right) \times \mathbb{T}$ and $\left(r_{n}, \infty\right) \times \mathbb{T}$. Let $k_{0}$ be the 
value that appears in Theorem 4. If $|k| \geq k_{0}$ and $r \in\left(0, r_{1}\right)$, then using representation (3.11), Minkowski's inequality, the form (3.10) of $\varphi_{k}$, and the uniform estimate (3.12), we obtain

$$
\begin{aligned}
& \left(\int_{0}^{r_{1}} \int_{-\pi}^{\pi}\left|\chi_{k, j}^{\prime \prime}(r) e^{i k \theta}\right|^{2} r \mathrm{~d} \theta \mathrm{d} r\right)^{1 / 2} \\
& \leq \sqrt{2 \pi} \sum_{q=1}^{n}\left|a_{j q}\right|\left(\int_{0}^{r_{q}}\left|\frac{\mathrm{d}^{2}}{\mathrm{~d} r^{2}}\left[\varphi_{k}\left(\frac{r}{r_{q}}\right)\right]\right|^{2} r \mathrm{~d} r\right)^{1 / 2} \\
& =\sqrt{\frac{\pi}{2}}\left(k^{2}-1\right) \sum_{q=1}^{n}\left|a_{j q}\right|\left(\int_{0}^{1}\left|t^{|k|-2}\left[|k|-(|k|+2) t^{2}\right]\right|^{2} t \mathrm{~d} t\right)^{1 / 2} \leq n \sqrt{2 \pi}|k|^{3 / 2}
\end{aligned}
$$

Hence, for any positive integers $m, p$, with $m \geq k_{0}$, a new application of Minkowski's inequality gives

$$
\begin{aligned}
& \left(\int_{0}^{r_{1}} \int_{-\pi}^{\pi}\left|\sum_{k=m}^{m+p} \sum_{j=1}^{n} \widehat{\mu}_{j, k} \chi_{k, j}^{\prime \prime}(r) e^{i k \theta}\right|^{2} r \mathrm{~d} \theta \mathrm{d} r\right)^{1 / 2} \\
& \quad \leq \sum_{k=m}^{m+p} \sum_{j=1}^{n}\left|\widehat{\mu}_{j, k}\right|\left(\int_{0}^{r_{1}} \int_{-\pi}^{\pi}\left|\chi_{k, j}^{\prime \prime}(r) e^{i k \theta}\right|^{2} r \mathrm{~d} \theta \mathrm{d} r\right)^{1 / 2} \\
& \quad \leq n \sqrt{2 \pi} \sum_{j=1}^{n} \sum_{k=m}^{m+p}\left|\widehat{\mu}_{j, k}\right||k|^{3 / 2} .
\end{aligned}
$$

Therefore, since $\mu_{j} \in W^{2}$, it follows that the Fourier expansion of $s_{r r}^{A}$ is indeed a Cauchy series in $L^{2}\left(\left(0, r_{1}\right) \times \mathbb{T}\right)$. It can be also verified that similar arguments deliver the same conclusion for the interval $\left(r_{n}, \infty\right)$ in place of $\left(0, r_{1}\right)$, as well as for the other two functions of (2.2), which proves that $s^{A} \in \mathcal{S}_{B L_{2}}(\rho)$.

In addition, as section 3 shows, any biharmonic BL polyspline from $\mathcal{S}_{B L_{2}}(\rho)$ satisfying the same interpolation conditions (2.5) as $S^{A}$ can be expanded in an absolutely convergent Fourier series in $\theta$ with coefficients from $\mathcal{S}_{k}(\rho)$, for $k \in \mathbb{Z}$. Since these coefficients interpolate the same data values as those of $s^{A}$, the uniqueness statement of Theorem 3 implies the uniqueness of $S^{A}$ with the properties required in part (a) of Theorem 1.

Note that similar arguments also apply to prove part (b), the main difference being that, for $k=0$, we replace $\widehat{s}_{0}^{A}$ by $\widehat{s}_{0}^{B} \in \mathcal{S}_{0}(\rho)$, such that $\widehat{s}_{0}^{B}\left(r_{j}\right)=\widehat{\mu}_{j, 0}$, $j \in\{1, \ldots, n\}$, and $\widehat{s}_{0}^{B} \in \operatorname{span}\left\{r^{2}, 1\right\}$ for $r \in\left(0, r_{1}\right)$, as in the last statement of Theorem 3 (ii). The corresponding series (4.1) now defines $s^{B} \in \mathcal{S}_{B L_{2}}(\rho)$, which verifies the transfinite interpolation conditions (2.6) and the additional condition of being biharmonic at the origin.

\section{Variational Characterization}

To prove Theorem 2, we need the following orthogonality result.

Theorem 5. Let $S \in \mathcal{S}_{B L_{2}}(\rho)$ and $F \in V_{2}$ such that $f\left(r_{j}, \theta\right)=0, \forall \theta \in \mathbb{T}$, $\forall j \in\{1, \ldots, n\}$. If, in addition, either $F(0)=0$, or $S$ is biharmonic at 0 , then

$$
\langle s, f\rangle_{V_{2}}=0 \text {. }
$$


Proof. For any $F \in V_{2}$, recall the notation (3.1) for the Fourier coefficients of $f$ with respect to $\theta$. We will employ the following Parseval relation, which holds for any $S$ and $F$ from $V_{2}$ :

$$
\frac{1}{2 \pi}\langle s, f\rangle_{V_{2}}=\sum_{k=-\infty}^{\infty} J_{k}
$$

where

$$
\begin{aligned}
J_{k}:= & \int_{0}^{\infty}\left\{\frac{\mathrm{d}^{2} \widehat{s}_{k}}{\mathrm{~d} r^{2}} \frac{\mathrm{d}^{2} \widehat{\widehat{f}}_{k}}{\mathrm{~d} r^{2}}+2 k^{2}\left[\frac{\widehat{s}_{k}}{r^{2}}-\frac{1}{r} \frac{\mathrm{d} \widehat{s}_{k}}{\mathrm{~d} r}\right]\left[\frac{\widehat{\hat{f}_{k}}}{r^{2}}-\frac{1}{r} \frac{\mathrm{d} \widehat{\widehat{f}}_{k}}{\mathrm{~d} r}\right]\right. \\
& \left.+\left[k^{2} \frac{\widehat{s}_{k}}{r^{2}}-\frac{1}{r} \frac{\mathrm{d} \widehat{s}_{k}}{\mathrm{~d} r}\right]\left[k^{2} \frac{\overline{\widehat{f}_{k}}}{r^{2}}-\frac{1}{r} \frac{\mathrm{d} \widehat{\widehat{f}}_{k}}{\mathrm{~d} r}\right]\right\} r \mathrm{~d} r .
\end{aligned}
$$

The validity of (5.2) follows from (3.2) by arguments similar to those needed to obtain Eq. (28) in [5, Lemma 3.5]. Next, letting

$$
I_{k}:=\int_{0}^{\infty} r^{3}\left[R_{k} \widehat{s}_{k}(r)\right]\left[R_{k} \overline{\widehat{f}_{k}}(r)\right] \mathrm{d} r, \quad k \in \mathbb{Z},
$$

where $R_{k}$ is the 'right-hand' operator defined in (3.4) and (3.5), we claim that

$$
J_{k}=I_{k}, \quad \forall k \in \mathbb{Z}
$$

Indeed, if $k=0$, then

$$
\begin{aligned}
I_{0}-J_{0} & =\int_{0}^{\infty}\left(\frac{\mathrm{d}^{2} \widehat{s}_{0}}{\mathrm{~d} r^{2}} \frac{\mathrm{d} \frac{\widehat{f}_{0}}{\mathrm{~d} r}}{\mathrm{~d} r}+\frac{\mathrm{d} \widehat{s}_{0}}{\mathrm{~d} r} \frac{\mathrm{d}^{2} \widehat{\widehat{f}}_{0}}{\mathrm{~d} r^{2}}\right) \mathrm{d} r=\int_{0}^{\infty} \frac{\mathrm{d}}{\mathrm{d} r}\left(\frac{\mathrm{d} \widehat{s}_{0}}{\mathrm{~d} r} \frac{\mathrm{d} \overline{\widehat{f}_{0}}}{\mathrm{~d} r}\right) \mathrm{d} r \\
& =\lim _{r \rightarrow \infty} \frac{\mathrm{d} \widehat{s}_{0}}{\mathrm{~d} r}(r) \frac{\mathrm{d} \widehat{\widehat{f}}_{0}}{\mathrm{~d} r}(r)-\lim _{r \rightarrow 0} \frac{\mathrm{d} \widehat{s}_{0}}{\mathrm{~d} r}(r) \frac{\mathrm{d} \overline{\widehat{f}_{0}}}{\mathrm{~d} r}(r) .
\end{aligned}
$$

Note that $\frac{\mathrm{d} \widehat{s}_{0}}{\mathrm{~d} r} \in \operatorname{span}\{r, r \ln r\}$ for $r \in\left(0, r_{0}\right)$, and $\frac{\mathrm{d} \widehat{s}_{0}}{\mathrm{~d} r} \in \operatorname{span}\left\{r^{-1}\right\}$ for $r>r_{n}$, so $\lim \frac{\mathrm{d} \widehat{s}_{0}}{\mathrm{~d} r}(r)=0$ as $r \rightarrow 0$ and as $r \rightarrow \infty$. Hence $I_{0}-J_{0}=0$ will follow if we show that $\frac{\mathrm{d} \widehat{f}_{0}}{\mathrm{~d} r}(r)=O(1)$, as $r \rightarrow 0$ and as $r \rightarrow \infty$. Since, by Lemma 2 , we have $r^{1 / 2} \frac{\mathrm{d}^{2} \widehat{f_{0}}}{\mathrm{~d} r^{2}}$ and $r^{-1 / 2} \frac{\mathrm{d} \widehat{f}_{0}}{\mathrm{~d} r} \in L^{2}(0, \infty),\left[3\right.$, Lemma 1] implies $\frac{\mathrm{d} \widehat{f}_{0}}{\mathrm{~d} r}(r)=O(1)$, as $r \rightarrow 0$. To obtain the same property as $r \rightarrow \infty$, we make the simplifying notation $\psi:=\widehat{f}_{0}$, then use the Newton-Leibniz formula

$$
r \psi^{\prime}(r)-\psi^{\prime}(1)=\int_{1}^{r}\left[t \psi^{\prime}(t)\right]^{\prime} \mathrm{d} t=\int_{1}^{r} t^{1 / 2}\left[t^{1 / 2} \psi^{\prime \prime}(t)+t^{-1 / 2} \psi^{\prime}(t)\right] \mathrm{d} t
$$

and the Cauchy-Schwarz estimate:

$$
\begin{aligned}
& \left|\int_{1}^{r} t^{1 / 2}\left[t^{1 / 2} \psi^{\prime \prime}(t)\right] \mathrm{d} t\right|+\left|\int_{1}^{r} t^{1 / 2}\left[t^{-1 / 2} \psi^{\prime}(t)\right] \mathrm{d} t\right| \\
& \quad \leq\left|\int_{1}^{r} t \mathrm{~d} t\right|^{1 / 2}\left\{\left.\left.\left|\int_{1}^{r}\right| t^{1 / 2} \psi^{\prime \prime}(t)\right|^{2} \mathrm{~d} t\right|^{1 / 2}+\left.\left.\left|\int_{1}^{r}\right| t^{-1 / 2} \psi^{\prime}(t)\right|^{2} \mathrm{~d} t\right|^{1 / 2}\right\} \\
& \quad \leq 2^{-1 / 2}\left|r^{2}-1\right|^{1 / 2}\left\{|| r^{1 / 2} \psi^{\prime \prime}\left\|_{L^{2}(0, \infty)}+\right\| r^{-1 / 2} \psi^{\prime} \|_{L^{2}(0, \infty)}\right\}
\end{aligned}
$$


We deduce $I_{0}-J_{0}=0$. If $k \neq 0$, we employ the notation:

$$
\begin{aligned}
& T_{1}(r):=r^{2} \frac{\mathrm{d}}{\mathrm{d} r}\left[\frac{1}{r} \widehat{s}_{k}(r)\right] \frac{\mathrm{d}}{\mathrm{d} r}\left[\frac{1}{r}{\widehat{\widehat{f}_{k}}}_{k}(r)\right], \quad T_{2}(r):=\frac{\mathrm{d} \widehat{s}_{k}}{\mathrm{~d} r}(r) \frac{\mathrm{d} \widehat{\widehat{f}}_{k}}{\mathrm{~d} r}(r), \\
& T_{3}(r):=\frac{1}{r^{2}} \widehat{s}_{k}(r) \overline{\widehat{f}_{k}}(r),
\end{aligned}
$$

and, after some algebraic manipulation, we obtain

$$
\begin{aligned}
I_{k}-J_{k}= & \left(2|k|-k^{2}\right) \int_{0}^{\infty}\left\{\left(\widehat{s}_{k}\right)^{\prime \prime}\left(\frac{\overline{\widehat{f}_{k}}}{r}\right)^{\prime}+\left(\frac{\widehat{s}_{k}}{r}\right)^{\prime}\left(\overline{\widehat{f}_{k}}\right)^{\prime \prime}-2\left(\frac{\widehat{s}_{k}}{r}\right)^{\prime}\left(\frac{\widehat{\widehat{f}_{k}}}{r}\right)^{\prime}\right\} r \mathrm{~d} r \\
& +\left(k^{2}-1\right) \int_{0}^{\infty}\left[\left(\widehat{s}_{k}\right)^{\prime \prime}\left(\overline{\widehat{f}_{k}}\right)^{\prime}+\left(\widehat{s}_{k}\right)^{\prime}\left(\widehat{\widehat{f}_{k}}\right)^{\prime \prime}\right] \mathrm{d} r \\
& +2|k|\left(k^{2}-1\right) \int_{0}^{\infty}\left\{\left(\frac{1}{r} \widehat{s}_{k}\right)\left(\frac{1}{r} \overline{\widehat{f}_{k}}\right)^{\prime}+\left(\frac{1}{r} \widehat{s}_{k}\right)^{\prime}\left(\frac{1}{r} \widehat{\widehat{f}_{k}}\right)\right\} \mathrm{d} r \\
= & \int_{0}^{\infty}\left[\left(2|k|-k^{2}\right) T_{1}(r)+\left(k^{2}-1\right) T_{2}(r)+2|k|\left(k^{2}-1\right) T_{3}(r)\right]^{\prime} \mathrm{d} r \\
= & {\left[\left(2|k|-k^{2}\right) T_{1}(r)+\left(k^{2}-1\right) T_{2}(r)+2|k|\left(k^{2}-1\right) T_{3}(r)\right]_{r=0}^{r=\infty} . }
\end{aligned}
$$

If $|k|=1$, we only need to evaluate $\lim T_{1}(r)$ as $r \rightarrow 0$ and as $r \rightarrow \infty$. Since $\widehat{s}_{k} \in \mathcal{S}_{k}(\rho)$, in this case there exist constants $c_{1}, c_{2}$, such that $\left[\frac{1}{r} \widehat{s}_{k}(r)\right]^{\prime}=c_{1} r$ for $r \in\left(0, r_{0}\right)$, while $\left[\frac{1}{r} \widehat{s}_{k}(r)\right]^{\prime}=c_{2} r^{-3}$ for $r>r_{n}$. On the other hand, [6, Lemma 1 (ii)] implies, either as $r \rightarrow 0$ or as $r \rightarrow \infty$,

$$
r^{2}\left[\frac{1}{r} \widehat{f}_{k}(r)\right]^{\prime}=r\left(\widehat{f}_{k}\right)^{\prime}(r)-\widehat{f}_{k}(r)=O\left(r|\ln r|^{1 / 2}\right) .
$$

We deduce $\lim T_{1}(r)=0$ either as $r \rightarrow 0$ or as $r \rightarrow \infty$, hence $I_{k}-J_{k}=0$ for $k= \pm 1$.

If $|k| \geq 2$, we use the fact that $\widehat{s}_{k} \in \operatorname{span}\left\{r^{|k|+2}, r^{|k|}\right\}$ for $r \in\left(0, r_{0}\right)$, and $\widehat{s}_{k} \in \operatorname{span}\left\{r^{-|k|+2}, r^{-|k|}\right\}$ for $r>r_{n}$, together with [6, Lemma 1 (i)] to obtain $T_{l}(r)=O(r)$ as $r \rightarrow 0$ for $l \in\{1,2,3\}$, as well as $T_{l}(r)=O\left(r^{-1 / 2}\right)$ as $r \rightarrow \infty$ for $l \in\{1,3\}$, and $T_{2}(r)=O\left(r^{-3 / 2}\right)$ as $r \rightarrow 0$. Therefore $I_{k}-J_{k}=0$ holds in this case, also.

To conclude the proof, we invoke the orthogonality results for Beppo Levi $L_{k}$-splines [6, Theorem 1] and [3, Theorem 1], which show that $I_{k}=0, \forall k \in \mathbb{Z}$. Hence (5.2) and (5.3) imply (5.1).

Proof of Theorem 2. This is a standard consequence of Theorem 5: replacing $f$ in (5.1) by the current $f-s$, we obtain $\langle s, f-s\rangle_{V_{2}}=0$, which implies $0 \leq\|f-s\|_{V_{2}}^{2}=\|f\|_{V_{2}}^{2}-\|s\|_{V_{2}}^{2}$. Further, if $\|f\|_{V_{2}}=\|s\|_{V_{2}}$, then $f-s$ must be in the null space of the seminorm (2.3), which is equivalent to

$$
(f-s)(r, \theta)=r\left(c_{1} \sin \theta+c_{2} \cos \theta\right)+c_{3}, \quad \forall r>0, \forall \theta \in \mathbb{T} .
$$

Since the transfinite interpolation conditions imply $(f-s)\left(r_{j}, \theta\right)=0$ for $j \in$ $\{1, \ldots, n\}$ and all $\theta \in \mathbb{T}$, we deduce $f-s \equiv 0$, which completes the proof. 


\section{Acknowledgement}

I thank the referee for several suggestions which helped to improve the presentation.

\section{References}

[1] R.S. Al-Sahli. $L$-spline interpolation and biharmonic polysplines on annuli. Master's thesis, Kuwait University, Department of Mathematics, 2012.

[2] R. Arcangéli, M.C. López de Silanes and J.J. Torrens. Multidimensional Minimizing Splines. Theory and Applications. Kluwer, Boston, 2004.

[3] A. Bejancu. Radially symmetric thin plate splines interpolating a circular contour map. Preprint, Kuwait University, 2013.

[4] A. Bejancu. Semi-cardinal polyspline interpolation with Beppo Levi boundary conditions. J. Approx. Theory, 155:52-73, 2008. http://dx.doi.org/10.1016/j.jat.2008.04.010.

[5] A. Bejancu. Transfinite thin plate spline interpolation. Constr. Approx., 34:237256, 2011. http://dx.doi.org/10.1007/s00365-010-9118-3.

[6] A. Bejancu and R.S. Al-Sahli. A new class of interpolatory $L$-splines with adjoint end conditions. Preprint, Kuwait University, 2013.

[7] A. Böttcher and B. Silbermann. Introduction to Large Truncated Toeplitz Matrices. Springer, New York, 1998.

[8] J. Duchon. Interpolation des fonctions de deux variables suivant le principe de la flexion des plaques minces. RAIRO Anal. Numer., 10:5-12, 1976.

[9] G.H. Golub and C.F. Van Loan. Matrix Computations. Third ed. The Johns Hopkins University Press, Baltimore, 1996.

[10] D.R. Jenkins. Thin plate spline interpolation on an annulus. ANZIAM J., 42:819-836, 2000.

[11] J. Jerome and J. Pierce. On spline functions determined by singular self-adjoint differential operators. J. Approx. Theory, 5:15-40, 1972. http://dx.doi.org/10.1016/0021-9045(72)90027-5.

[12] M.J. Johnson. Compactly supported, piecewise polyharmonic radial functions with prescribed regularity. Constr. Approx., 35:201-223, 2012.

http://dx.doi.org/10.1007/s00365-011-9141-z.

[13] O.I. Kounchev. Multivariate Polysplines. Applications to Numerical and Wavelet Analysis. Academic Press, London, 2001.

[14] T.R. Lucas. A generalization of L-splines. Numer. Math., 15:359-370, 1970. http://dx.doi.org/10.1007/BF02165507.

[15] C. Rabut. Interpolation with radially symmetric thin plate splines. J. Comput. Appl. Math., 73:241-256, 1996. http://dx.doi.org/10.1016/0377-0427(96)00046-5.

[16] M.A. Sabin. Transfinite surface interpolation. In G. Mullineux(Ed.), The Mathematics of Surfaces VI, volume 58 of IMA Conf. Ser., New Ser., pp. 517-534. Oxford University Press, 1996.

[17] L.L. Schumaker. Spline Functions: Basic Theory. Third ed. Cambridge University Press, 2007.

[18] H. Wendland. Scattered Data Approximation. Cambridge University Press, Cambridge, 2005. 